\title{
Entre espoir et désespoir
}

L'enseignement de la citoyenneté dans les écoles indiennes

Between hope and despair, Citizenship education in Indian schools

Entre esperanza y desespero,La educación ciudadana en las escuelas indias

\section{D.S. Muley}

Traducteur : Robert Elbaz

\section{(2) OpenEdition}

\section{Journals}

Édition électronique

URL : https://journals.openedition.org/ries/171

DOI : 10.4000/ries. 171

ISSN : 2261-4265

\section{Éditeur}

France Education international

Édition imprimée

Date de publication : 1 avril 2007

Pagination : 105-113

ISBN : 978-2-85420-569-5

ISSN : 1254-4590

\section{Référence électronique}

D.S. Muley, «Entre espoir et désespoir », Revue internationale d'éducation de Sèvres [En ligne], 44 | avril 2007, mis en ligne le 23 juin 2011, consulté le 09 juillet 2021. URL : http://journals.openedition.org/ ries/171 ; DOI : https://doi.org/10.4000/ries.171 


\title{
Entre espoir et désespoir
}

L'enseignement de la citoyenneté dans les écoles indiennes*

\author{
D. S. Muley
}

Dans le contexte indien, la réponse à toute question, y compris celle portant sur le statut de l'enseignement de la citoyenneté, est toujours ambivalente. La taille gargantuesque de l'Inde, sa diversité confondante en matière de langues et de religions, sa classe moyenne en expansion associée à une poignée de gens extrêmement riches contrastant avec une masse illettrée de pauvres, auxquelles il faut ajouter, pour couronner le tout, une multiplicité de soussystèmes éducatifs et de types d'établissements - tout cela rend impossible toute question sur l'Inde considérée de façon globale.

\section{La Politique nationale D'ENSEIGNEMENT (1986): LE TRONC COMMUN}

Cela posé, il semble judicieux de lancer le débat par une citation tirée de la Politique nationale d'enseignement (1986). "Le système national d'éducation sera fondé sur un ensemble structuré de programmes nationaux qui contiennent un tronc commun. Ce tronc commun comprendra l'histoire de la libération de l'Inde, les obligations établies par la Constitution et tout autre contenu de nature à fortifier le sentiment d'identité nationale. Ces éléments fonctionneront de façon transdisciplinaire et seront conçus de manière à promouvoir des valeurs telles que l'héritage commun de l'Inde, l'égalitarisme, la démocratie et la laïcité, l'égalité des sexes, la protection de l'environnement, l'abolition des barrières sociales, le respect de la limitation des naissances et le développement de la culture scientifique. » Dès 1952, la commission de l'enseignement secondaire a défini la citoyenneté comme concept englobant des qualités intellectuelles, sociales et morales. L'Inde a choisi d'être une république démocratique et laïque. Comme telle, tout citoyen doit avoir l'esprit critique nécessaire pour distinguer les faits de la propagande et pour rejeter les sirènes du fanatisme et des préjugés. De même, ce même citoyen ne devrait pas condamner ce qui est ancien sous le prétexte que c'est ancien - ou accepter ce qui est nouveau simplement parce que c'est nouveau. Bien plutôt, tout citoyen devrait examiner en toute objectivité les aspects contradictoires et rejeter fermement ce qui est contraire à l'équité, à la justice et au développement.

\footnotetext{
${ }^{*}$ Article traduit par Robert Elbaz.
} 
Toute la question est maintenant de savoir si nous préparons nos enfants d'âge scolaire à devenir des citoyens jouissant de ces idéaux exigeants et de ces qualités élevées.

\section{LA SALLE DE CLASSE ET LA RÉALITÉ SOCIALE}

Toutes les grandes questions qui se font jour, tant à l'échelle nationale qu'internationale, ont été incorporées aux programmes scolaires, depuis la préservation de l'environnement, les droits de l'homme, la paix et la compréhension internationales, le communautarisme et le fondamentalisme, l'éducation aux valeurs, la justice et l'égalité entre sexes, jusqu'à la prolifération du SIDA. Un coup d'œil rapide aux programmes scolaires indiens montre qu'aujourd'hui, les enfants bénéficient de toutes les informations utiles concernant ces sujets. L'approche qui en est faite est certes scolaire, bien que des efforts récents aient été consentis pour associer ce savoir scolaire à la réalité socioculturelle. On ne saurait toutefois nier que le savoir dispensé au sein de la classe est encore dans une large mesure coupé des réalités sociales.

Un article sur le sens civique en Inde, publié le 26 novembre 2006 dans le quotidien The Times of India fournit des détails presque graphiques du sens civique et de l'étiquette qui prévalent en Inde : "Si les Jeux Olympiques reconnaissaient comme sports officiels le rot, le crachat, le fait de jeter des détritus dans les parcs publics et dans la rue ou de se soulager en public, les Indiens auraient rapporté une moisson de médailles d'or!», bien que certaines fonctions corporelles aient une justification socioculturelle. Par exemple, roter revient à exprimer sa satisfaction, tant vis-à-vis du repas servi que vis-à-vis de ses hôtes. Les autres fonctions corporelles se voient expliquées par la pauvreté et l'ignorance endémiques, associées à une migration massive des villages vers les villes. Dans ce contexte, il est encourageant de constater que «les enfants d'âge scolaire font preuve de davantage de sens civique que les adultes.»

\section{LES "DEVOIRS FOndamentaUX " DÉFINIS PAR LA CONSTITUTION}

Il est important de garder en mémoire que les éléments du tronc commun mentionnés dans la Politique nationale d'éducation de 1986 et dans la révision entreprise en 1992 trouvent leur origine dans les Devoirs fondamentaux contenus dans la Constitution indienne. Cet article met particulièrement l'accent sur cet aspect parce que les dix Devoirs fondamentaux ainsi que les éléments figurant dans le tronc commun sont au centre même du concept d'éducation à la citoyenneté en Inde.

Les Devoirs fondamentaux contenus dans l'article 51A ont été incorporés à la Constitution indienne lors de la promulgation du $42^{\mathrm{e}}$ amendement à 
la Constitution, en 1976. Suivant l'article 51A, il est du devoir de chaque citoyen indien de :

- respecter la Constitution, ses idéaux et institutions, le drapeau national et l'hymne national;

- chérir et suivre les nobles idéaux qui ont inspiré la lutte de notre pays pour la liberté;

- soutenir et protéger la souveraineté, l'unité et l'intégrité de l'Inde;

- défendre la nation et s'acquitter de tout devoir national lorsque cela est nécessaire;

- promouvoir l'harmonie et l'esprit de fraternité de tous les Indiens, par delà les diversités religieuses, linguistiques, régionales ou particulières ; renoncer aux pratiques portant atteinte à la dignité des femmes;

- mettre en valeur et préserver le riche héritage de notre culture plurielle;

- protéger et améliorer l'environnement et la nature - forêts, lacs, fleuves, vie sauvage - et faire preuve de compassion à l'égard de toute forme de vie ;

- développer l'esprit scientifique, les valeurs humanistes et l'esprit de questionnement et de réforme;

- protéger le bien public et abjurer toute forme de violence;

- s'efforcer de tendre vers l'excellence dans tous les aspects des activités individuelles et collectives, de manière à élever sans cesse la nation vers un niveau supérieur d'effort et de réalisation.

À la fin des années 1990, une évaluation a été conduite par le gouvernement indien et le ministère du Développement des ressources humaines, sur ordre de la Cour suprême de l'Inde, pour analyser le contenu des programmes d'enseignement des écoles et des enseignants, à la lumière des Devoirs fondamentaux mentionnées dans la Constitution. Cette évaluation trouve son origine dans une lettre de l'ancien procureur général Ranganath Misra, datée du 18 mars 1998 et adressée au procureur général actuel, qui a été considérée comme un véritable acte d'accusation. Dans cette lettre, l'auteur déclarait que «nous faisons tous avec horreur l'expérience quotidienne de comportements dégradants en société. Cette dégradation ne cesse de s'accentuer et ne saurait s'améliorer, à moins de porter un coup d'arrêt immédiat à cette chute (...). Les Devoirs fondamentaux sont restés lettre morte au sein de la Constitution et n'en sont jamais sortis - fût-ce au bénéfice de ceux qui la rédigent.» Ce haut magistrat a insisté sur le fait qu' "une mesure de nature à rebâtir la nation - enseigner les Devoirs fondamentaux dans chaque institution éducative» est indispensable pour qu'un équilibre satisfaisant entre droits et devoirs puisse se faire jour. Dans ce contexte, je ne résiste pas à la tentation de rappeler l'existence de la Déclaration universelle des obligations de l'homme, proposée par l'InterAction Council (Conseil des actions internationales), un groupe d'experts de haut niveau, lors de la réunion qui s'est tenue à Vienne du 20 au 22 avril 1997. Nous avons jusqu'à présent toujours fait référence aux droits fondamentaux de 
l'homme comme faisant partie intégrante de l'héritage du mouvement de libération nationale qui a abouti à l'indépendance de notre pays en 1947. Il est temps aujourd'hui de parler de ses responsabilités.

L'analyse des programmes scolaires entreprise par le National Council of Educational Research and Training (Conseil national de la recherche et de la formation pédagogiques) révèle que les idéaux et les principes ambitieux contenus dans les Droits fondamentaux sont repris de façon satisfaisante dans le programme de différentes matières, principalement en sciences sociales (éducation civique/politique et histoire), en langues et en sciences. Parmi les concepts et les contenus liés à la citoyenneté qui trouvent leur place dans les programmes scolaires à tous les niveaux, l'on trouve les caractéristiques fondamentales de la Constitution indienne, ses principes de démocratie, de laïcité et d'unité dans la diversité, les droits fondamentaux du citoyen, les droits de l'homme, les défis économiques et sociaux auxquels l'Inde et la société indienne doivent faire face, ainsi que la notion de développement économique et social.

Dans la réalité de la pratique pédagogique au sein de la classe, l'essentiel des contenus et des questions associés à cette thématique est traité de manière incidente. Rares sont les points qui font l'objet de débats directs et approfondis. Les activités collectives des établissements se limitent la plupart du temps au respect des fêtes religieuses et des journées commémoratives nationales, à des activités culturelles, à l'affichage des décisions de la direction de l'établissement, aux assemblées matinales et autres activités communautaires de service. On peut de surcroît préciser que quelques-unes de ces activités collectives s'avèrent n'être que de simples rituels qui, par leur mode d'organisation même, n'inspirent que rarement les élèves, pas plus qu'ils n'influencent leur comportement et leurs valeurs.

\section{LES CONTRAINTES D'UN SYSTÈME FONDÉ SUR L'ÉVALUATION}

La nature de l'enseignement à la citoyenneté fait qu'il appartient fondamentalement au domaine non cognitif. La citoyenneté démocratique ne se limite pas au statut légal du citoyen et au droit de vote. Elle inclut tous les aspects de la vie dans une société démocratique. L'éducation à la citoyenneté donne à chaque élève les connaissances, les aptitudes et les comportements nécessaires pour jouer un rôle actif dans la société, comme citoyens informés, socialement et moralement responsables. Elle cherche à leur donner la confiance et la conviction nécessaires pour œuvrer avec d'autres au bien commun et à établir une vraie différence dans leur vie de citoyens. L'éducation à la citoyenneté consiste à préparer des individus à participer, comme citoyens actifs et responsables, au fonctionnement démocratique de la société. Grâce à elle, il s'agit de déterminer qui nous sommes, comment vivre ensemble, quels adultes nos enfants deviendront, ce qui en fait un concept éminemment normatif. 
Le système scolaire en Inde repose sur l'évaluation et les examens. Seule la connaissance des contenus et des informations est évaluée. Ce qui n’est pas évalué lors des examens annuels n'est pas enseigné en classe. En situation pédagogique réelle, l'accent est mis sur les informations et les contenus plutôt que sur les aptitudes plus souples, telles que le comportement ou les valeurs. On pourrait donc faire valoir qu'il ne suffit pas d'incorporer ces idéaux et ces principes aux programmes scolaires pour atteindre l'objectif fixé. Puisque la question d'inculquer aux élèves des valeurs et un comportement civiques est liée au débat plus vaste de la qualité de l'enseignement et de l'apprentissage en Inde, l'enseignement de l'éducation à la citoyenneté ne devrait pas être considéré de façon séparée.

\section{L'ABSENCE DE TOUTE STRUCTURE D'ENCOURAGEMENT}

La façon dont l'éducation à la citoyenneté peut s'avérer contreproductive sans l'existence d'une structure d'encouragement socioculturelle peut être illustrée par un exemple pris en Inde où $30 \%$ de la population vit avec moins d'un dollar par jour. Le professeur Sen Varma, responsable de la validité des élections en Inde dans les années soixante et lui-même enseignant, avait pensé à éduquer les électeurs ordinaires que les candidats cherchaient à acheter pour obtenir leurs votes. Il fit préparer de grands panneaux, sur lesquels on pouvait lire : «Votre vote a de la valeur : n'acceptez pas de le vendre.» Un jeune au chômage aperçut le panneau et lui dit : «Merci, professeur. Je ne savais pas que c'était possible.»

La vie civique et la vie politique en Inde sont si inextricablement liées que l'on ne trouve que très peu de personnes actives dans leur vie civique qui ne soient, directement ou indirectement, impliquées dans la vie politique. De plus, la perception que le public a des hommes politiques a atteint une sorte de nadir ${ }^{1}$. Le citoyen lambda perçoit la politique comme un cloaque. L'expression «personne n'est au-dessus des lois» est devenue un cliché en Inde. Le nombre considérable de membres du Parlement ayant des casiers judiciaires donne l'impression que l'on a bien plutôt affaire à une cour des miracles. Mais les jugements récents rendus par les tribunaux, dans lesquels certaines personnes très influentes et puissantes, ainsi que leurs fils, ont été condamnées à de la prison ferme semblent indiquer une tendance selon laquelle lesdits puissants se voient maintenant contraints de rendre compte de leurs crimes, tant mineurs que graves. La criminalisation de la politique altère quelque peu le lustre de la politique en Inde. On estime à 700 (sur un total de 4 072) les parlementaires qui ont des antécédents judiciaires; 120 sont députés de la Lok Sabha (Chambre basse).

1. Ndt : point virtuel situé à l'opposé du zénith. 
Le taux de condamnation est très bas et le temps d'incarcération varie de dix à vingt ans. Dans une situation semblable, il serait presqu'impossible à un homme ordinaire, aux moyens modestes, d'obtenir un procès équitable ou de faire appel. Mais le système démocratique indien a fait la preuve de sa résilience et a su mettre en place des contre-feux sous la forme d'un système judiciaire vigilant, d'une presse et de médias pugnaces et d'une classe moyenne éduquée qui ne cesse de croître. La plus grande démocratie du monde a fait taire tous les oiseaux de mauvais augure.

Cela nous conduit à une autre observation importante faite par Fareed $Z_{\text {Zakaria }}^{2}$ qui déclare que la démocratie tire un profit immense de la plupart de ses traits «non-démocratiques». La Cour suprême indienne et ses juges qui siègent sans avoir été élus ont joué un rôle remarquable au cours des dernières années dans la définition et le rétablissement de valeurs citoyennes importantes.

Selon Transparency International ${ }^{3}$, l'Inde est passée du $88^{\mathrm{e}}$ au $70^{\mathrm{e}}$ rang mondial dans la lutte contre la corruption, bien que plus de $60 \%$ des participants à une étude conduite par Transparency India aient déclaré que la corruption faisait partie intégrante de leur vie. Cependant, grâce à l'avènement de l'e-gouvernance et de la loi relative aux droits de l'information, la situation s'améliore. «Les gens ordinaires paient en Inde un tribut annuel estimé à deux milliards de roupies ${ }^{4}$ (soit environ 35000 euros) en bakchichs pour différents services liés à la police, au système judiciaire, à la gestion du territoire, aux municipalités et à la santé. ${ }^{5}$

Nous constatons en fait que l'ossature constitutionnelle actuelle de l'Inde est résolument démocratique et libérale, alors que la société elle-même n'est pas encore au diapason de cette ossature. Dans son style inimitable, Fareed Zakaria ajoute : «(...) à maints égards, (le pays) est devenu plus démocratique. Mais il est aussi devenu moins tolérant, moins laïque, moins respectueux des lois, moins libéral. Ces deux tendances - la démocratisation et l'intolérance ${ }^{6}-$ sont directement liées.»

L'Inde demeure néanmoins un exemple éclatant, non seulement en Asie, mais dans le monde entier, de la manière dont la démocratie est parvenue, avec succès sinon avec efficacité, à perdurer pendant plus d'un demi-siècle, contre vents et marées.

C'est dans cette réalité, mêlant messages optimistes et pessimistes, que les élèves doivent pénétrer pour devenir des citoyens à part entière. L'éducation à la citoyenneté dispensée par l'école ne les prépare guère à gérer de telles

\footnotetext{
2. Fareed Zakaria, Daniel Roche : L'avenir de la liberté. La démocratie illibérale aux États-Unis et dans le monde, Odile Jacob, 2003.

3. Transparency International est une ONG dont le siège est à Berlin et qui a pour objet de combattre et prévenir la corruption dans les transactions internationales.

4. 1 euro équivaut à 57200 roupies environ (janvier 2007).

5. The Times of India, ${ }_{1}^{\mathrm{er}}$ janvier 2007.

6. Ndt : intolérance traduit ici le terme «illiberalism».
} 
contradictions. Le défi que doit relever l'éducation civique dans les écoles aujourd'hui est d'inculquer des qualités citoyennes telles que l'honnêteté, l'intégrité, le respect des lois, la volonté de faire valoir ses droits légitimes, doublée d'une volonté en tous points égale de respecter ses obligations légales et morales. Ce défi est doublement accru par le fait que les exemples d'individus malhonnêtes dont les affaires prospèrent et embellissent sont loin d'être l'exception. Par moments, le tableau semble fort sombre. À d'autres moments, il semble au contraire que le problème prend des proportions sans commune mesure avec la réalité.

Le principe fondamental de l'apprentissage est que les élèves doivent découvrir et explorer les concepts et les comportements qui mènent à une citoyenneté saine par eux-mêmes. La technique de la clarification des valeurs, prônée par tout un chacun aujourd'hui, ne transmet aucun ensemble de valeurs particulières. Au lieu de cela, elle aide les élèves à identifier les valeurs auxquelles ils adhèrent et celles qu'ils développent, et leur permet en outre d'agir sur leurs propres valeurs, avec conviction et avec cohérence. L'accent est davantage mis sur le processus que sur le résultat. Dans les classes des écoles indiennes, c'est généralement l'inverse. Or le processus et le résultat doivent être harmonieusement combinés pour inculquer aux élèves les valeurs civiques.

Avec la technique de la clarification des valeurs, l'enseignant est censé créer un cadre de vie démocratique dans lequel les élèves devraient pouvoir dire ce qu'ils ressentent, et non ce qu'ils pensent que leurs enseignants ont envie d'entendre. Les aptitudes des enseignants à jouer un tel rôle sont amoindries par les différentes limitations qu'ils rencontrent. Leur formation, tant durant leurs études qu'au cours de leur vie professionnelle, n'a elle aussi qu'un impact limité sur leurs aptitudes. Le nombre moyen des élèves dans une classe indienne (cinquante environ, mais ce chiffre peut localement monter jusqu'à quatrevingt ou quatre-vingt-dix), les consignes insistantes du système pour que les enseignants limitent leur enseignement aux manuels et à leur contenu, l'évaluation des enseignants sur la seule base des résultats obtenus par leurs élèves aux examens - tout cela fait que même les enseignants les plus inspirés ne sont guère motivés pour dépasser le cadre de la simple transmission des informations et des contenus.

Dans un tel contexte, les enseignants ne semblent disposer que d'un espace extrêmement limité pour créer l'occasion d'inculquer à leurs élèves des valeurs citoyennes. Des efforts sont faits pour mettre en place une réforme des examens, afin de promouvoir l'apprentissage dans des domaines extrascolaires. L'une de ces réformes consiste à introduire le système dit d'évaluation continue globale (Continuous and Comprehensive Evaluation, CCE), ou évaluation d'établissement. Le Conseil des directions d'établissements (Council of Boards of School Education, COBSE), une association de quarante-et-une équipes de direction dans tout le pays, a fait des efforts, à sa modeste échelle, pour mettre en 
place un système opérationnel de CCE dans lequel les résultats des élèves dans des domaines extrascolaires, tels que l'éducation civique, pourraient également être évalués, motivant ainsi les enseignants pour tenter d'inculquer aux élèves les valeurs civiques tant désirées.

\section{QUELQUES SIGNES ENCOURAGEANTS}

Ponctuellement, des élèves ont fait preuve d'une extraordinaire conscience civique qui a laissé une trace durable. Un exemple illustre cela. Diwali est une fête importante en Inde, comparable à Noël dans le monde occidental. Durant Diwali, l'une des pratiques les plus répandues et les plus populaires parmi les enfants est l'utilisation de pétards chez soi. Il y a environ dix ans, la ville de Delhi suffoquait littéralement tant la fumée de ces pétards était épaisse. Puis, il y a quelques années, les enfants d'âge scolaire ont décidé de limiter - faute de pouvoir totalement le contrôler - l'usage des pétards durant Diwali, afin de préserver la qualité de l'air. La différence fut nettement perceptible, grâce à cette décision écologique prise volontairement par ces enfants. Tout en mentionnant cette trouée d'espoir, je suis douloureusement conscient que de tels signes encourageants sont circonscrits à des métropoles telles que Delhi et sont fort rares ailleurs.

L'aptitude à tolérer les différentes opinions et croyances, qui est la marque de la citoyenneté démocratique, a été de tous temps l'un des traits saillants de la tradition et de la culture indiennes. La citoyenneté démocratique exige d'être mise en pratique dans la vie. Cette pratique suppose de développer les aptitudes des élèves et d'influencer leur comportement. Dans ce contexte, le projet de concours du Parlement de la jeunesse, initié il y a une trentaine d'années par le ministère des Affaires parlementaires et destiné aux élèves et aux étudiants, mérite une mention particulière ici. Plus de vingt-deux États du pays offrent l'occasion à ces jeunes de prendre part au programme, tant au niveau de chaque état qu'au niveau national. Ce programme de Parlement de la jeunesse est d'un grand intérêt pédagogique car il contribue à enraciner chez les jeunes les qualités nécessaires à une citoyenneté harmonieuse.

En matière d'enseignement scolaire formel, la contribution des organisations non gouvernementales $(\mathrm{ONG})$, et tout spécialement des organisations à but non lucratif, n'a pas été reconnue à sa juste valeur en Inde. Les ONG qui opèrent dans le domaine de l'éducation ont amélioré les connaissances du grand public et fourni une éducation aux jeunes. Leur travail, tout spécialement auprès des enfants déscolarisés, dont le nombre est considérable en Inde, est digne d'éloges. Contrairement aux agences gouvernementales, ces ONG ont l'avantage de disposer d'une méthodologie innovante et souple. Depuis peu, des efforts lucides ont été faits pour les associer à la mise au point des programmes et à la conception des 
manuels au niveau national. Il est réconfortant de voir qu'un nombre non négligeable de jeunes ayant brillamment réussi s'inspire des ONG et de leur travail.

Dans l'affaire du meurtre spectaculaire d'un jeune mannequin, survenu dans un café, le principal suspect, le fils d'un homme politique influent et puissant, a été mis hors de cause par un tribunal. Il s'en est sorti en menaçant ou en achetant les témoins. Cet acquittement a suscité un choc dans tout le pays et un profond sentiment de révolte et d'injustice. De jeunes Indiens, au nombre desquels figuraient des étudiants activement soutenus par la presse et les médias électroniques, se sont dressés comme un seul homme contre ce verdict et ont ébranlé le système judiciaire anesthésié. Moins d'un an plus tard, la Haute Cour a annulé le verdict et condamné l'assassin à la réclusion criminelle à perpétuité. Ajoutons qu'un certain nombre de films indiens, qui ont une influence non négligeable sur les jeunes esprits, ont également fait preuve du même esprit civique et de la même action en faveur de la justice.

Il est cependant difficile d'évaluer la part que l'éducation civique dispensée dans les écoles et à l'université joue dans ces exemples édifiants.

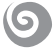

Cet article se proposait de mettre en lumière le versant citoyen de l'enseignement scolaire en termes d'espoir et de désespoir. Au terme du bilan, il semble que le premier l'emporte sur le second.

\section{BIBLIOGRAPHIE}

AMARTYA SEN (2005): The Argumentative Indian, London, Penguin Group.

Citizenship Development and Fundamental Duties (1988), New Delhi, Citizenship Development Society.

FAREED Zakaria (2003): The Future of Freedom, New Delhi, Penguin Books India.

Interim Report of The Committee Set Up By The Government Of India, Operationalisation of the Suggestions to Teach Fundamental Duties to the Citizens of the Country (January 30, 1999), New Delhi, NCERT.

MULEY D.S. (1999): Conducting Youth parliament, New Delhi, NCERT.

National Curriculum Framework 2005, New Delhi, National Council of Educational Research and Training.

National Policy on Education 1986, New Delhi, Ministry of Human Resource development, Government of India. 